\title{
可逆空间的连通支不必可逆
}

\author{
陈吉 象 \\ (南开大学数学系, 天溹)
}

Rajagopalan 和 Wilansky 在文献[1]中提出了可逆拓扑空间的概念, 此后一些作者也做 了一系列的研究. 对任意拓扑空间 $X$, 令 $E(X)$ 和 $H(X)$ 分别表示 $X$ 到自身的连续双射 (即既单又满的连续映射)和自同胚的全体. 如果 $E(X)-H(X)$, 则 $X$ 称为可逆拓扑空间, 否则称 $X$ 为非可逆的. 可逆空间包括了紧致 Hausdorff 空间以及 $n$ 维(对一彴正整数 $n$ )不带 边流形等一大类空间. 文献 [1]定理 6 指出, 若 $X$ 由有限个连通支组成, 则 $X$ 可逆的充要条件 是每个连通支均为可逆. 并且由可数无穷个可逆连通支组成的空间不必可逆. 但文献 [1] 没 有说及一般可逆空间的连通支是否可逆. 本文的目的是给出一个例子, 说明可逆空间的连通 支不必是可逆的. 当然, 根据文献 [1] 的定理 5, 局部连通可逆空间的每个连通支必为可逆 的.

引王 若 $A$ 是可逆空间 $X$ 的子空间,且任意 $f \in E(A)$ 可扩张为 $g \in E(X)$, 则 $A$ 也是 可逆的.

推论 设 $X$ 为非可逆空间,则拓扑锥形 $C X$ (即商空间 $X \times I / X \times\{0\}$,其中 $I$ 为单位 闭区间 $[0,1])$ 为非可逆的.

为便于叙述要构造的例子,我们在三维欧氏空间 $\mathbf{R}^{3}$ 中采用柱面坐标,即 $(r, \theta, z)$. 文 献[1]已指出实数空间 $R^{1}$ 的有理数全体 $Q$ 为非可逆的. 现定义一个同胚 $\mathbf{R}^{1} \cong\langle 0,1\rangle-\{x$ $\left.\in \mathbf{R}^{1} \mid 0<x<1\right\}$ 为

$$
X \longmapsto \frac{1}{2}+\frac{1}{2} \cdot \frac{x}{1+|x|}, \quad x \in \mathbf{R}^{1},
$$

它诱导同胚 $Q \cong\langle 0,1\rangle \cap Q$. 令 $S^{2}$ 是单位圆

$$
S^{1}-\left\{(1, \theta, 0) \in \mathbf{R}^{3} \mid 0 \leqslant \theta \leqslant 2 x\right\} \text {. }
$$

定义指数映射 $\langle 0,1\rangle \rightarrow S^{\prime}$ 为

$$
\iota \longmapsto(1,2 \pi t, 0), \quad t \in\langle 0,1\rangle,
$$

它诱导同胚 $\langle 0,1\rangle \cap \mathrm{Q} \cong A \subset S^{1}$. 我们把 $A$ 的点称为 $S^{1}$ 的“有理点”, $S^{1}-A$ 的点称为非有 理点, 因可逆性是拓扑不变的, 故 $A$ 为非可逆的. 令 $C_{0}$ 是以 $e$ 为顶点, $A$ 为底的拓扑锥形 $C A$, 则由推论知, $C$ 。为非可逆的. 其次令

$$
C_{n}-\left\{\left(1, \theta, \frac{1}{n}\right) ; 0 \leqslant \theta \leqslant 2 \pi\right\}, n-1,2, \cdots,
$$

$Y=4 \cup\left(\bigcup_{n=1}^{\infty} C_{n}\right) \subset \mathbf{R}^{3}$. 而我们要构造的例子是商空间

$$
X-(Y+C A) / a \sim p(a, 1),
$$

本文1986年9月15日收到. 
其中“+”表示拓扑和, $p: A \times I \longrightarrow C A$ 是粘合映射, $a$ 取遍 $A$ 中的点. 显然, 每 $-C_{n}(n=$ $0,1,2, \cdots)$ 是 $X$ 的连通支. 我们将说明 $X$ 是可逆的.

设 $f \in E(X)$. 我们指出 $f$ 的下列性质:

(1) $f\left(C_{0}\right)=C_{0}$, 且对任意正整数 $k$, 存在正整数 $f(k)$, 使得 $\left.\bar{f}\right|_{c_{k}^{k}}: C_{k} \cong C_{f(k)}$.

事实上，因 $C_{k} \cong S^{1}$ 是连通紧致 1 维流形，不能嵌人 $C_{0}$ 中. 因此对 $k>0$, 存在 $\bar{f}(k)>$

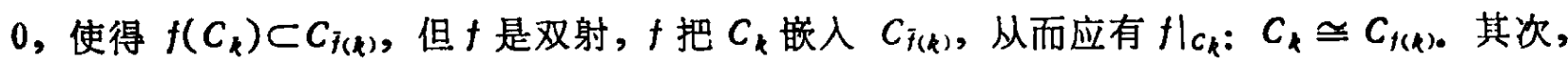
$f$ 是满射, 应有 $f\left(C_{0}\right)-C_{0}$. 同时还可知, 在 $\bar{f}$ 下, $k \mapsto \bar{f}(k)$ 是正整数集合到自身的双射.

(2) $f(A)=A, f(e)=e$, 且对任意 $a \in A$, 有 $\left.f\right|_{[e, a]:}[e, a] \cong[e, f(a)]$, 其中

$$
[e, a]=p(\{a\} \times l) \text {. }
$$

事实上,对任意 $a \in A, C_{0}-\{a\}$ 道路连通,而对 $c \in C_{0}-A, C_{0}-\{c\}$ 不再道路连通, 由 $f$ 的连续性及 $f\left(C_{0}\right)=C_{0}$ 可知 $f(A) \subset A$. 其次, 如果 $f(e)=p\left(a_{0}, \lambda_{0}\right) \in C_{0}-\{e\}$, 对 某 $a_{0} \in A, 0<\lambda_{0} \leqslant 1$, 则取 $f(c)$ 在 $X$ 中的开邻域 $X-p\left(A \times\left[0, \lambda^{\prime}\right]\right), 0<\lambda^{\prime}<\lambda_{0}$, 使 得 $e \notin X-p\left(A \times\left[0, \lambda^{\prime}\right\rangle\right)$. 然后在 $C A$ 中有 $e$ 的道路连通开邻域 $B-p\left(\bigcup_{a \in A}\left(U_{a} \times\left\{0, \varepsilon_{a}\right\rangle\right)\right)$, 其中 $U_{a}$ 是 $a$ 在 $A$ 中的开邻域, $0<\varepsilon_{a}<1$, 使得 $f(B) \subset X-p\left(A \times\left[0, \lambda^{\prime}\right]\right)$. 于是 $f(B) \subset$ $p\left(\left\{a_{0}\right\} \times\langle 0,1]\right)=\left\langle e, a_{\varphi}\right\}$. 因 $f$ 是单射, 这将导致矛盾, 故 $f(e)=e$. 然后不难说明, 对任 意 $a \in A$, 我们有 $\left.f\right|_{[e, a]}:[e, a] \cong[e, f(a)]$. 进而推出 $f(A)=A$.

为叙述 $f$ 的进一步性质,规定 $S^{1}$ 的定向为右定向, 即 $(r, \theta)$ 平面上的逆时针方向, 同时规 定每个 $C_{k}(k>0)$ 的相应定向. 对 $S^{1}$ 的相异有序三点组 $(a, b, c)$, 如果向量 $\boldsymbol{a b}$ 与 $\boldsymbol{a c}$ 的 向量积 $\boldsymbol{a b} \times \boldsymbol{a c}$ 的方向与 $\boldsymbol{z}$ 轴正向一致,我们就说 $(a, b, c)$ 是顺向组,否则就说 $(a, b, c)$ 是逆向组. 对 $C_{k}(k>0)$ 及 $A$ 中的相异有序三点组相应地规定顺向或逆向. 类似地, 对于 相异有序三点组 $(\alpha, \beta, \gamma)$, 使得 $\alpha \in\langle e, a], \beta \in\langle e, b\rfloor, \gamma \in\langle e, c]$, 其中 $a, b, c$ 为 $A$ 中相 异点, 如果 $(a, b, c)$ 是顺向组, 就说 $(\alpha, \beta, \gamma)$ 是顺向组, 否则就说 $(\alpha, \beta, \gamma)$ 是逆向组. 现在可以叙述 $f$ 的下述性质.

(3) 对 $C_{0}-\{e\}$ 中任意顺向组 $\left(a_{1}, \alpha_{2}, \alpha_{3}\right)$, 其 $f$ 象 $\left(f\left(\alpha_{1}\right), f\left(\alpha_{2}\right), f\left(\alpha_{3}\right)\right)$ 也是顺向 组.

由性质 (2) 只需考虑 $\alpha_{i} \in A$ 的情形, $i=1,2,3$. 假定不是这样, 即有 $A$ 中的一个顺向组 $\left(\alpha_{1}, \alpha_{2}, \alpha_{3}\right)$, 使得 $\left(f\left(\alpha_{1}\right), f\left(\alpha_{2}\right), f\left(\alpha_{3}\right)\right)$ 是逆向组. 我们说明此时对 $A$ 中任意顺向组 ( $\alpha_{1}^{\prime}$, $\left.\alpha_{2}^{\prime}, \alpha_{3}^{\prime}\right),\left(f\left(\alpha_{1}^{\prime}\right), f\left(\alpha_{2}^{\prime}\right), f\left(\alpha_{3}^{\prime}\right)\right)$ 将均为逆向组. 事实上, 设 $\alpha_{i}-\left(1, \theta_{i}, 0\right) \in A, i-1,2,3$, 不妨设 $0<\theta_{1}<\theta_{2}<\theta_{3}<2 \pi$. 考虑 $\mathrm{Y}$ 中三个点列

$$
u_{\text {in }}-\left(1, \theta_{i}, \frac{1}{n}\right), n-1,2, \cdots, i-1,2,3,
$$

显然 $\lim _{n \rightarrow \infty} u_{i n}=a_{i}$, 由连续性, $\lim _{n \rightarrow \infty} f\left(u_{i n}\right)-f\left(\alpha_{i}\right), i=1,2,3 .\left(f\left(\alpha_{1}\right), f\left(a_{2}\right), f\left(a_{3}\right)\right)$ 是逆向 组. 不难看出, 存在正整数 $N$, 使得 $n>N$ 时, $\left(f\left(u_{1 n}\right), f\left(u_{2 n}\right), f\left(u_{3 n}\right)\right)$ 都是逆向组. 又因 $\left.f\right|_{C_{n}}: C_{n} \cong C_{7(n)}$, 因此, 对 $n>N$ 及 $C_{n}$ 中任意一个顺向组 $\left(u_{1}^{\prime}, u_{2}^{\prime}, u_{3}^{\prime}\right),\left(f\left(u_{1}^{\prime}\right), f\left(u_{2}^{\prime}\right), f\left(u_{3}^{\prime}\right)\right)$ 是 $C_{\bar{f}(n)}$ 中的逆向组. 现设 $\left(\alpha_{1}^{\prime}, \alpha_{2}^{\prime}, \alpha_{3}^{\prime}\right)$ 是 $A$ 中任意一个顺向组, 与上述相仿,利用点列的极 限可以说明 $\left(f\left(\alpha_{1}^{\prime}\right), f\left(\alpha_{2}^{\prime}\right), f\left(\alpha_{3}^{\prime}\right)\right)$ 是逆向组. 然后, 当 $f$ 已经出现上述这种情况时, 我们就定 义同胚 $\boldsymbol{S}^{1} \rightarrow \boldsymbol{S}^{1}$ 为

$$
(1, \theta, 0) \mapsto(1,2 \pi-\theta, 0), \theta \in[0,2 \pi],
$$


这个同胚显然可诱导一个同泼 $h \in H(X)$, 使得

$$
\begin{gathered}
h(e)-e, \\
h\left(1, \theta, \frac{1}{n}\right)-\left(1,2 \pi-\theta, \frac{1}{n}\right), n-1,2, \cdots, \\
h p((1, \theta, 0), \lambda)-p((1,2 \pi-\theta, 0), \lambda),(1, \theta, 0) \in A, 0<\lambda \leqslant 1 .
\end{gathered}
$$

然后用 $h f \in E(X)$ 代替原来的 $f$, 则 $h f$ 具有性质(3)如果能证明 $h f \in H(X)$, 则立刻有 $f \in$ $H(X)$. 因此总可以假定 $f$ 具有性质(3).

(4) $f^{-1} \mid y: Y \rightarrow Y$ 连续.

首先, 对任意 $n>0, C_{n}$ 是 $Y$ 的开集, 根据(1), 不难说明 $f^{-1} \mid y$ 在每一 $C_{n}$ 连续, 从而 在 $\bigcup_{n=1}^{\infty} C_{n}$ 连续. 其次, 设 $a^{\prime} \in A, f^{-1}\left(a^{\prime}\right)-a \in A$, 考虑 $a$ 在 $Y$ 中的任意形如 $(\widehat{\xi \eta} \times[0,8\rangle)$ $n Y-U$. 的开邻域, 其中 $\xi, \eta$ 是 $S^{1}$ 的两个非有理点, 使得 $(\xi, a, \eta)$ 是顺向组, 且

$$
\widehat{\xi \eta}-\left\{x \in S^{1} \mid(\xi, x, \eta) \text { 是顺向组 }\right\} \text {, }
$$

8 是任意小的正数. 再取 $\xi_{1}, \eta_{1} \in \widehat{\xi \eta} \cap A$, 使得 $\left(\xi_{1}, a, \eta_{1},\right)$ 是顺向组. 令 $\overline{\xi_{1} \eta_{1}}-\widehat{\xi_{1} \eta_{1}} \cup\left\{\xi_{1}\right.$, $\left.\eta_{1}\right\}$. 记 $U_{n}-\left(\overline{\xi_{1} \eta_{1}} \times[0,8)\right) \cap C_{n}$, 对 $n>\frac{1}{8}$. 若 $\xi_{1}-\left(1, \theta_{1}^{\prime}, 0\right), \eta_{1}-\left(1, \phi_{1}^{\prime}, 0\right)$, 则有 极限

$$
\lim _{n \rightarrow \infty} f\left(1, \theta_{1}^{\prime}, \frac{1}{n}\right)-f\left(\xi_{1}\right), \lim _{n \rightarrow \infty} f\left(1, \psi_{1}^{\prime}, \frac{1}{n}\right)-f\left(\eta_{1}\right),
$$

使得 $\left(f\left(\xi_{1}\right), a^{\prime}, f\left(\eta_{1}\right)\right)$ 是顺向组. 因 $\left(S^{1} \times[0,8\rangle\right) \cap Y$ 外仅有有限个连通支 $C_{n}$, 故有正整 数 $M$, 使得 $n>M$ 时, 恒有 $f^{-1}\left(C_{n}\right) \subset\left(S^{1} \times[0,8)\right) \cap Y$. 由此不难得到 $a^{\prime}$ 在 $Y$ 中的邻域 $U_{a^{\prime}}$, 使得 $f^{-1}\left(U_{a^{\prime}}\right) \subset U_{a}$, 因此证明了 $\left.f^{-1}\right|_{Y}$ 在 $a^{\prime} \in A$ 连续, 即有 $\left.f^{-1}\right|_{\mathrm{Y}}: Y \rightarrow Y$ 连续.

(5) $f^{-1} c_{0}: C_{0} \rightarrow C_{0}$ 连续.

为此定义 $7, f^{-1}: A \times I \rightarrow A \times I$ 为

$$
\begin{gathered}
f(a, 0)-(f(a), 0), f^{-1}(a, 0)=\left(f^{-1}(a), 0\right), a \in A, \\
f(a, \lambda)=p^{-1} f p(a, \lambda), f^{-1}(a, \lambda)=p^{-1} f^{-1} p(a, \lambda), a \in A, \lambda \in\langle 0,1],
\end{gathered}
$$

显然 $f$ 在 $A \times\langle 0,1]$ 内连续. 由此再根据 $f$ 的性质(2)及(3)可以证明 $7^{-1}: A \times I \rightarrow A \times$ $I$ 连续. '最后因 $p f^{-1}-f^{-1} p: A \times l \rightarrow C_{0},\left.f^{-1}\right|_{c_{0}}: C_{0} \rightarrow C_{0}$ 是 $p f^{-1}$ 诱导的, 于是 $\left.t^{-1}\right|_{0}$ : $C_{0} \rightarrow C_{0}$. 连续.

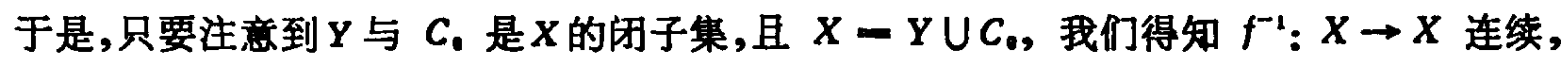
即 $f \in H(X), X$ 可逆.

\section{劣 文}

[i] Rajagopalan, M. and Wilensky, A., Reversible topological spaces, J. Austral. Math. Soc., 61 (1966), $129-138$. 